\title{
Utility of the Polestar N30 low-field MRI system for resecting non-enhancing intra-axial brain lesions
}

\author{
Lior Ungar*, Zion Zibly*, Anton Wohl, Ran Harel, Moshe Hadani, Moshe Attia, \\ Roberto Spiegelmann, Zeev Feldman, Jacob Zaubermann, Nachshon Knoller, Zvi R. Cohen \\ Department of Neurosurgery, Sheba Medical Centre, Sackler School of Medicine, Tel Aviv University, Tel Aviv, Israel
}

\begin{abstract}
Background. To determine the utility of an intraoperative magnetic resonance imaging (iMRI) system, the Polestar N30, for enhancing the resection control of non-enhancing intra-axial brain lesions.

Materials and methods. Seventy-three patients (60 males [83.3\%], mean age 37 years) with intra-axial brain lesions underwent resection at Sheba Medical Centre using the Polestar between February 2012 and the end of August 2018. Demographic and imaging data were retrospectively analysed. Thirty-five patients had a non-enhancing lesion (48\%).

Results. Complete resection was planned for 60/73 cases after preoperative imaging. Complete resection was achieved in 59/60 (98.3\%) cases. After iMRI, additional resection was performed in 24/73 (32.8\%) cases, and complete resection was performed in $17 / 60$ (28.8\%) cases in which a complete resection was intended. In $6 / 13(46 \%)$ patients for whom incomplete resection was intended, further resection was performed. The extent of resection was extended mainly for non-enhancing lesions: 16/35 (46\%) as opposed to only $8 / 38(21 \%)$ for enhancing lesions. Further resection was not significantly associated with sex, age, intended resection, recurrence, or affected side. Univariate analysis revealed non-eloquent area, intended complete resection, and enhancing lesions to be predictive factors for complete resection, and non-enhancing lesions and scan time to be predictive factors for an extended resection. Non-enhancement was the only independent factor for extended resection.

Conclusions. The Polestar N30 is useful for evaluating residual non-enhancing intra-axial brain lesions and achieving maximal resection.
\end{abstract}

Key words: glioma, image guided surgery, MRI, surgical management

(Neurol Neurochir Pol 2021; 55 (2): 202-211)

\section{Introduction}

The Polestar system (Medtronic, Louisville, CO, USA) is a low-field ( 0.15 Tesla) intraoperative magnetic resonance imaging (iMRI) system, introduced in 2001 by our group at the Sheba Medical Centre in Tel-Aviv [1]. This system comprises an MRI scanner integrated with an optical tracking system. The system is installed in a conventional radiofrequency-shielded operating room. When the scanner is placed under the operating room table, the magnetic field strength in the surgical field is low, enabling the use of standard surgical equipment.
From 1999 to 2018, a total of 236 patients with intra-axial brain lesions were operated on using three generations of the Polestar iMRI (N10, N20, and N30).

In our previous experience including a cohort of $163 \mathrm{pa}-$ tients operated on between 1999 and 2012 [2], we concluded that non-enhancing lesions were the only independent variable that predicted further resection using the iMRI system. As a result of that study, we changed our policy and almost half of the patients operated on between February 2012 and August 2018 had non-enhancing brain lesions, compared to only one third of the patients in the previous cohort.

Address for correspondence: Zvi R. Cohen, Department of Neurosurgery, Sheba Medical Centre, Sackler School of Medicine, Tel Aviv University, Tel Aviv, Israel, e-mail: zvi.cohen@sheba.health.gov.il

*These authors contributed equally to this study 
Table 1. Pathology characteristics

\begin{tabular}{|c|c|c|c|}
\hline Pathology & $\begin{array}{c}\text { Number of cases } \\
n=73\end{array}$ & $\begin{array}{l}\text { Non enhancing } \\
\quad n=35\end{array}$ & $\begin{array}{l}\text { Enhancing } \\
\qquad n=38\end{array}$ \\
\hline AA WHO III & $8(11)$ & $6(17.1)$ & $2(5.3)$ \\
\hline AO WHO III & $12(16.4)$ & $4(11.4)$ & $8(21.1)$ \\
\hline Cavernoma & $3(4.1)$ & $0(0)$ & $3(7.9)$ \\
\hline Demyelinative & $1(1.4)$ & $0(0)$ & $1(2.6)$ \\
\hline Enhancing glial tumor WHO II & $1(1.4)$ & $0(0)$ & $1(2.6)$ \\
\hline Ependymoma WHO II & $1(1.4)$ & $0(0)$. & $1(2.6)$ \\
\hline Ependymoma WHO III & $1(1.4)$ & $0(0)$. & $1(2.6)$ \\
\hline Ganglioglioma WHOI & $1(1.4)$ & $0(0)$ & $1(2.6)$ \\
\hline GBM WHO IV & $5(6.8)$ & $0(0)$ & $5(13.2)$ \\
\hline Glioneuronal WHOI & $1(1.4)$ & $0(0)$. & $1(2.6)$ \\
\hline Gray matter heterotopia & $1(1.4)$ & $1(2.9)$ & $0(0)$ \\
\hline HGG WHO III & $4(5.5)$ & $2(5.7)$ & $2(5.3)$ \\
\hline JPA WHOI & $6(8.2)$ & $0(0)$ & $6(15.8)$ \\
\hline LGA WHO ॥ & $10(13.7)$ & $10(28.6)$ & $0(0)$ \\
\hline LGOWHO II & $12(16.4)$ & $11(31.4)$ & $1(2.6)$ \\
\hline MET & $1(1.4)$ & $0(0)$ & $1(2.6)$ \\
\hline Neurocytoma WHO I & $1(1.4)$ & $0(0)$ & $1(2.6)$ \\
\hline PNET WHO IV & $1(1.4)$ & $0(0)$ & $1(2.6)$ \\
\hline PXAWHOI & $2(2.7)$ & $0(0)$ & $2(5.3)$ \\
\hline Sub Ependymoma WHO I & $1(1.4)$ & $1(2.9)$ & $0(0)$ \\
\hline \multicolumn{4}{|l|}{${ }^{*}$ Grouping pathology } \\
\hline HGG WHO III-IV & $30(41)$ & $12(34)$ & $18(47)$ \\
\hline Miscellaneous & $21(29)$ & $2(6)$ & $19(50)$ \\
\hline LGG WHO ॥ & $22(30)$ & $21(60)$ & $1(3) p<0.001$ \\
\hline
\end{tabular}

*Grouping pathology: $p<0.001$; HGG WHO III-IV: AA, AO, Ependymoma III, GBM, HGG;

Miscellaneous: Cavernoma, Demyelinative, Enhncing, Ependymoma II, Ganglioglioma, Glioneuronal I, Gray matter., JPA, MET, Neurocytoma II, PNET, PXA, Subependymoma; LGG WHO II: LGO, LGA

In the current cohort of 73 patients using the third generation Polestar N30, we operated on very few patients with metastasis, and more often on patients with low-grade glioma than on patients with high-grade glioma. In addition, $12 / 30$ patients with a high-grade glioma had non-enhancing lesions (Tab. 1).

For the purpose of the current analysis, our goal was to compare the impact of iMRI for the surgical strategy and course of brain tumour resection in different types of lesions including non-enhancing and enhancing ones.

The survival benefits of extensive resection in patients with high-grade glioma are well established [3-8], whereas a limited number of studies have reported the survival benefits of extensive resection in patients with low-grade gliomas [9-11] A review of the current literature supporting safe, maximal resection for gliomas was recently provided by D’Amico et al. [12].

Based on our new policy and the efforts of the neurosurgical community to define the clinical benefits of safe, maximal tumour resection with respect to symptomatic relief and improved quality of life, progression-free survival and overall survival in patients having low-grade and high-grade glioma with molecular heterogeneity, we evaluated the feasibility of low-field MRI using the Polestar N30 to achieve extended resection with intraoperative neurophysiological monitoring (IONM) when needed, especially for non-enhancing brain lesions (both low-grade and high-grade gliomas), and we discuss its future role in an era of other advanced intraoperative technologies.

\section{Materials and methods}

\section{Patients}

Surgery was performed in 73 patients with intra-axial lesions using an intraoperative ultra-low-field MRI (Polestar N30, Medtronic) from February 2012 until the end of August 2018, in the Department of Neurosurgery, Sheba Medical Centre, Tel-Aviv, Israel. A total of 8/73 (11.1\%) patients underwent an awake craniotomy due to the proximity of the lesion to eloquent speech areas, and 25/73 (34\%) patients underwent 
resection under motor cortical and/or subcortical stimulation using magnetic resonance-compatible needles (Technomed Europe Medical Accessories, The Netherlands). Twenty-five patients (34\%) were operated on for recurrent tumours.

\section{Imaging and surgical methods}

Preoperative scans were performed in each patient using the Polestar N30 system in the operating room and then uploaded onto an image-guided navigation system (StealthStation, Medtronic). Resection was performed until the neurosurgeon felt he had achieved maximal resection or until the neurosurgeon felt that neurological stability was at risk. At this time, further intraoperative resection control scans were acquired. If a residual tumour was suspected on the resection control images, the surgeon explored the lesion so as to ascertain whether further resection was required. If the neurosurgeon felt that it was, additional resection control scans were obtained.

The intent of the resection was determined on the basis of preoperative images and functional MRI, and classified as either an intended complete or an incomplete lesion resection. Lesions located adjacent to or in eloquent brain areas, for which we believed an aggressive gross total resection might result in a substantial neurological deficit, were characterised as an intended incomplete resection. For the remaining lesions, the intent was to perform a complete resection. During the resection procedure, control images were obtained and compared to the preoperative iMRI images and the diagnostic images. The success of the resection was categorised as 'a complete resection' if no residual tumour was detected on one or more resection control images, or as an incomplete resection' if a residual tumour was observed.

Residual tumour for enhancing lesions was defined as the presence of nodular enhancement on the basis of T1-weighted images with gadolinium. Linear enhancement or a fuzzy enhancing lesion outside the area of the preoperative enhancing lesion was not considered a residual tumour but was instead defined as a complete resection. Residual tumour for non-enhancing lesions was based on the presence of a nodular or bulky lesion on FLAIR (fluid-attenuated inversion recovery) or T2-weighted images. Areas of FLAIR or T2-weighted image abnormality outside the area of the preoperative non-enhancing lesion were considered surgical procedure-induced changes and defined as a complete resection. (Supplemental Fig. 1-3) Diagnostic Flair and T1-weighted images with gadolinium are presented as well (Supplemental Fig. 1E-F) to gain understanding of the character, quality and resolution of the intraoperative scans.

A retrospective analysis of demographic data, diagnostic imaging, and pathology was performed. The data was retrieved from the patient's personal records and from our institute's imaging and pathology database. Additional data, such as the positioning of the patient, number of scans, sessions, and various time parameters, was obtained from the Polestar system database.

Finally, comparisons were made between the two success groups (i.e. complete and incomplete resection) and between cases in which iMRI led to further resection and those in which iMRI did not lead to further resection.

\section{Statistical methods}

Data was analysed with SPSS software version 25.0 (SPSS Inc., Chicago, IL, USA). Pathology characteristics according to enhancing/non-enhancing status were presented as frequencies and percentages for categorical variables. Chi-square tests and independent t-tests were performed to compare the two groups: complete/incomplete resection, and 2+ sessions for categorical and continuous variables, respectively. A multivariate analysis (Wald test, logistic regression) was performed, including factors such as age, sex, and enhancement. Hosmer-Lemeshow goodness-of-fit test was performed to assess the overall model. The significance level was set to less than 0.05 .

This study was approved by the institutional review board of Sheba Medical Centre.

\section{Results}

\section{Histological classification}

The pathologies were classified into three groups (Tab. 1): high-grade gliomas (World Health Organisation [WHO] grade III-IV $=30$ patients. These 30 comprised 12 anaplastic oligondendrogliomas [WHO III], eight anaplastic astrocytomas [WHO III], four high-grade glioma [WHO III], five glioblastoma multiforme [WHO IV], and one anaplastic ependymoma [WHO III]; low-grade gliomas (WHO II $=22$ patients. These 22 comprised 10 low-grade astrocytomas [WHO II] and 12 low-grade oligodendrogliomas [WHO II]), plus various other lesions (21 patients; three cavernous angiomas, one primitive neuroectodermal tumour, six pilocytic astrocytomas, one ependymoma, one demyelinative tumour, one ganglioglioma, one glioneuronal tumour, two pleomorphic xanthoastrocytomas, one central neurocytoma, one grey matter heterotopia, one subependymoma, one metastasis, and one enhancing glial tumour).

\section{Imaging characteristics}

Thirty-seven (51\%) of the lesions were right-sided, and $36(49 \%)$ were left-sided. The lesions were further divided according to their location: 45 (62\%) lesions were frontal, six $(8 \%)$ were temporal, $10(14 \%)$ were parietal, and the remaining 12 (16\%) were located in other areas (insular cortex, cerebellum, intraventricular occipital, or a combination of two lobes). During surgery, 55 (75\%) patients with mainly frontal and parietal lesions were positioned with their face upward (head-up position), six (8\%) patients with mainly occipital and cerebellar lesions were positioned in the head-down position, 
Table 2. Parameters in relation to the outcome

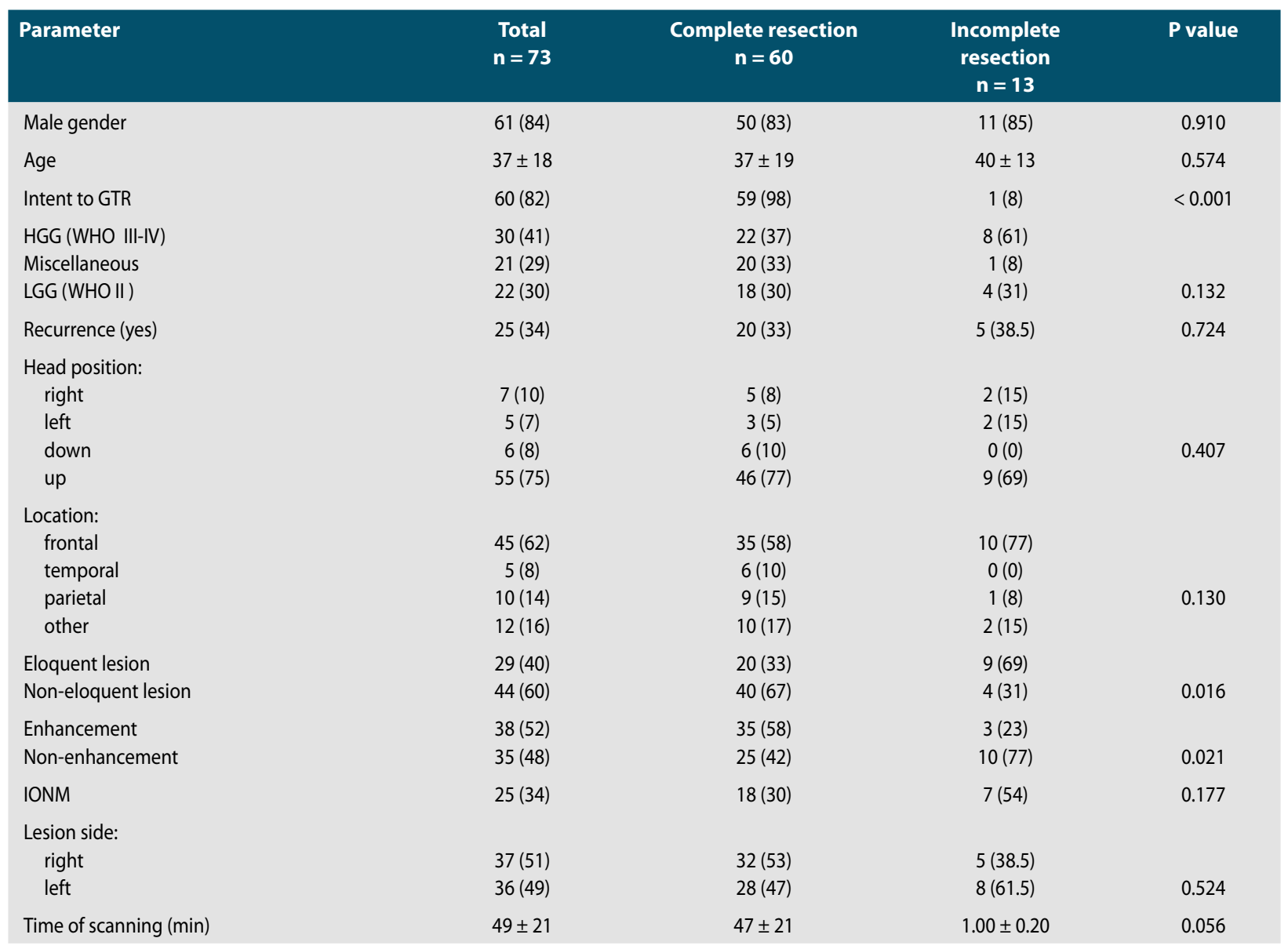

GTR - gross total resection, Eloquent lesion — adjacent to motor and speech centers, IONM — Intraoperative Neurophysiological Monitoring, HGG — High Grade Glioma, LGG — Low Grade Glioma

five $(7 \%)$ patients were operated on in a left side-up position, and seven $(10 \%)$ patients were operated on in a right side-up position. Twenty-nine (40\%) of the lesions were located in eloquent areas (adjacent to motor and speech centers) and 44 (60\%) were located in non-eloquent brain areas.

The radiology parameters were documented for each patient and included 38 contrast-enhancing lesions (52\%). The remaining 35 (48\%) lesions were non-enhancing lesions.

\section{Resection intent}

Among the 73 patients, the surgical intent was to perform complete tumour removal in $60(82.2 \%)$. For the remaining 13 patients, the surgical intent was to perform incomplete tumour removal. Of the 60 cases for which the surgical intent was to perform a complete resection, 59 (98.3\%) cases achieved a complete resection. In this subgroup of 59 patients, additional scans were performed in 17/59 (28.8\%) to increase the extent of the resection and to achieve complete resection. The surgical intent of the resection significantly correlated with the results ( $p<0.001$; Tab. 2$)$.

Of the 13 patients for whom the surgical intent was incomplete resection, 12 had an incomplete resection and one (7.7\%) had a complete resection. No additional scan was performed in this $13^{\text {th }}$ patient, but additional scans were performed in $6 / 12(50 \%)$ patients to increase the extent of the resection, even though incomplete resection was achieved (Fig. 1).

\section{Benefit of iMRI}

73 resections were performed with intent to achieve either complete or incomplete surgical resection. Among these 73 patients, iMRI led to further resection in 24 cases, indicating that iMRI led to a maximal tumour resection in $32.8 \%$ of the total cases without consideration of the outcome (complete/ /incomplete removal) (Fig. 1).

In 60 patients, the initial intent was to achieve complete lesion resection. In the remaining 13 patients, the surgical intent was to perform incomplete tumour removal. A complete resection was achieved in 60 patients ( 59 from the intent to perform complete resection group and one from the intent to perform incomplete resection group). The surgical intent of the resection significantly correlated with the results $(\mathrm{p}<0.001$; Tab. 2).

In $49(67.2 \%)$ of these patients, only two scan sessions were performed: a preoperative image acquisition within the operation room prior to surgery, and an intraoperative resection control image. These 49 patients account for the cases in which the surgeon did not perform additional resection 


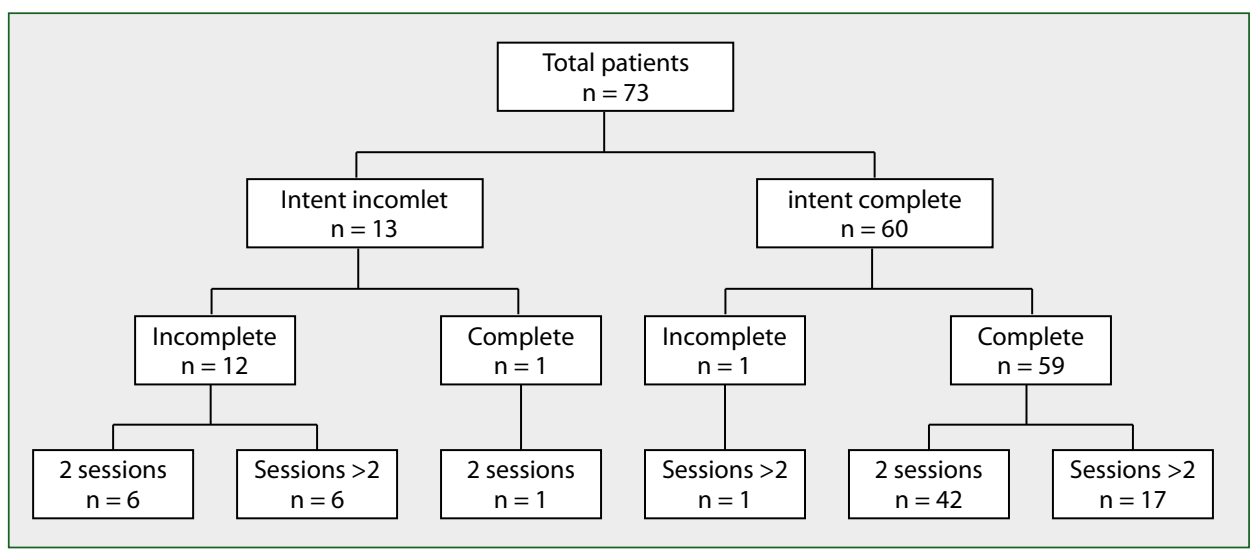

Figure 1. Distribution of cases in relation to intent of resection and number of sessions performed for resection control

after iMRI; 43 patients due to the absence of residual tumour observed on imaging, and six patients on the basis of the surgeon's decision not to proceed with the resection because further resection might compromise neurological function. More than two scan sessions were performed in 24 patients, and additional resection was performed due to the presence of residual tumour that was revealed by the resection control imaging. Complete resection was achieved in 18 of these patients. Thus, iMRI was responsible for $30.5 \%$ of the complete resections achieved in our study (in which the intent was to perform complete removal). Of these 24 patients, three imaging sessions were performed in 18 cases, indicating that in the large majority (75\%) of cases in which iMRI led to additional resection, two resection control images (a total of three scans, including the preoperative scan) were sufficient to achieve complete resection. In two cases, four sessions were performed, in one case, five sessions, and in one case, six sessions (Tab. 3).

Among the 13 patients for whom the initial intent was to perform an incomplete resection, incomplete tumour removal was achieved in 12 cases. In six of these cases, more than two scan sessions took place, indicating that iMRI led to further surgery to maximise the resection in $46.1 \%$ of this group. In one case, iMRI led to complete resection (following two scans), although the original intent was for incomplete resection.

In summary, the Polestar N30 iMRI system maximised the extent of the intra-axial lesion resection in $32.8 \%$ of all operations performed in this cohort. Use of the iMRI led to 17 additional complete lesion resections, representing a 40.6\% increase in complete lesion resections.

Interestingly, iMRI largely influenced the extent of the resection in cases in which the intent was incomplete resection, and was thus responsible for additional resection in $46.1 \%$ of patients in this group (Fig. 1).

Table 2 shows the relation between various parameters examined and the surgical outcome (resection success rates).

Age, sex, and diagnosis had no significant effect on resection success rates. Data regarding radiological characteristics,
Table 3. Distribution of cases in relation to number of iMRI scan sessions. More than 2 sessions indicates cases in which iMRI led to further resection

\begin{tabular}{|lc|}
$\begin{array}{l}\text { Number of scan } \\
\text { sessions per patient }\end{array}$ & Number of patients \\
\hline 2 & $49(67 \%)$ \\
3 & $18(25 \%)$ \\
4 & $4(5.4 \%)$ \\
5 & $1(1.3 \%)$ \\
6 & $1(1.3 \%)$ \\
\hline
\end{tabular}

e.g. enhancing vs. non-enhancing lesions, had no effect on the general success of the resection. Frontal lesions and left hemispheric lesions were more frequent in cases resulting in incomplete resection (related to the proximity to eloquent areas), but this difference was not statistically significant. On the other hand, non-eloquent area $(\mathrm{p}=0.016)$ and enhancing lesions $(\mathrm{p}=0.02)$ were predictive factors for complete resection in univariate analysis (Tab. 2).

Table 4 shows the relation between the various parameters examined and extended resection.

The extent of the resection was expanded mainly for non-enhancing lesions: $16 / 35$ (46\%) compared to only $8 / 38(21 \%)$ of the enhancing lesions.

Univariate analysis performed to compare cases in which additional resections were performed following resection control imaging (24 cases) with patients in which no further resection was performed (13 cases) identified scan time and enhancement to be the only variables that differed significantly between the two groups (Tab. 4).

Patients with non-enhancing lesions more frequently comprised cases in which additional resection was performed and univariate analysis revealed a significantly increased scan time. On the other hand, sex, age, intent of resection, recurrence, diagnosis, patient positioning, and location of the lesion were not significantly different between these two groups. 
A multivariate analysis (logistic regression) was performed (Tab. 5), with factors such as age, sex, diagnosis, and enhancement as covariates. Enhancement was the only independent variable. The odds ratio for non-enhancing lesions adjusted for age and sex was 3.1, indicating that there was a three-fold greater probability that a non-enhancing lesion would require more than two sessions to achieve the maximal tumour resection.

Table 4. Parameters in relation to extent of resection

\begin{tabular}{|c|c|c|c|}
\hline Parameter & $\begin{array}{l}2 \text { Sessions } \\
n=49\end{array}$ & $\begin{array}{c}>2 \text { Sessions } \\
n=24\end{array}$ & $\begin{array}{c}P \\
\text { value }\end{array}$ \\
\hline Male gender & $42(86)$ & $19(79)$ & 0.478 \\
\hline Age (years) & $36 \pm 20$ & $39 \pm 13$ & 0.425 \\
\hline Intent to GTR & $41(84)$ & $19(79)$ & 0.636 \\
\hline Recurrence (yes) & $17(35)$ & $8(33)$ & 0.908 \\
\hline $\begin{array}{l}\text { Lesion side: } \\
\text { right } \\
\text { left }\end{array}$ & $\begin{array}{l}22(45) \\
27(55)\end{array}$ & $\begin{array}{l}15(62.5) \\
9(37.5)\end{array}$ & 0.099 \\
\hline $\begin{array}{l}\text { Location: } \\
\text { frontal } \\
\text { temporal } \\
\text { parietal } \\
\text { other }\end{array}$ & $\begin{array}{c}25(51) \\
6(12) \\
9(18) \\
9(18)\end{array}$ & $\begin{array}{c}20(83) \\
0(0) \\
1(4) \\
3(12.5)\end{array}$ & 0.119 \\
\hline $\begin{array}{l}\text { Eloquent lesion } \\
\text { Non-eloquent lesion }\end{array}$ & $\begin{array}{l}18(37) \\
31(63)\end{array}$ & $\begin{array}{l}11(46) \\
13(54)\end{array}$ & 0.455 \\
\hline $\begin{array}{l}\text { Enhancement } \\
\text { Non-Enhancement }\end{array}$ & $\begin{array}{l}30(61) \\
19(39)\end{array}$ & $\begin{array}{r}8(33) \\
16(67)\end{array}$ & 0.025 \\
\hline IONM & $15(30)$ & $10(41)$ & 0.157 \\
\hline $\begin{array}{l}\text { Head position: } \\
\text { right } \\
\text { left } \\
\text { down } \\
\text { up }\end{array}$ & $\begin{array}{c}6(12) \\
4(8) \\
6(12) \\
33(67)\end{array}$ & $\begin{array}{c}1(4) \\
1(4) \\
0(0) \\
22(92)\end{array}$ & 0.060 \\
\hline Complete resection & $42(86)$ & $18(75)$ & 0.261 \\
\hline Time of scanning ( $\mathrm{min}$ ) & $44 \pm 21$ & $60 \pm 19$ & 0.003 \\
\hline $\begin{array}{l}\text { HGG WHO III-IV } \\
\text { Miscellaneous }\end{array}$ & $\begin{array}{l}19(39) \\
17(35)\end{array}$ & $\begin{array}{l}11(46) \\
4(17)\end{array}$ & \\
\hline LGG WHO ॥ & $13(26)$ & $9(37)$ & 0.265 \\
\hline
\end{tabular}

GTR - gross total resection, Eloquent lesion - adjacent to motor and speech centers, IONM Intraoperative Neurophysiological Monitoring, HGG — High Grade Glioma, LGG — Low Grade Glioma

\section{Scan data and time}

Resection control was based on T1-weighted images in 38 (52\%) patients and T2-weighted images in 35 (48\%) patients. FLAIR sequences were used to assist in determining the success of the resection. In 49 patients, two scan sessions were performed: a preoperative session and a resection control session that did not lead to further resection. The resection control session was performed at the time that the surgeon felt maximal resection had been achieved, or at the point at which the surgeon felt further resection would jeopardise the patient's neurological stability. In 18 patients, three iMRI sessions were performed: one preoperative, a second that led to further resection, and a third followed by no further resection. In four cases, four scan sessions were performed; in one case, five scans were performed; and in one case, six scans were performed. In all cases, no further resection was performed after the last intraoperative scan.

Imaging duration was defined as the sum of all scan durations from all sessions. Surgery overhead was defined as the sum of all session durations, including time spent between scans. This parameter does not include time spent before the first scan for preparing and positioning the device, yet serves as an important indicator of the surgical duration while using the Polestar iMRI system. The mean surgery overhead for cases with two sessions was $44 \mathrm{~min}$. Cases in which further resection was performed (more than two sessions) had a mean surgery overhead of $60 \mathrm{~min}$.

Thus, iMRI extended the surgery time by a mean of $16 \mathrm{~min}$ in cases in which resection control images led to additional resection as opposed to cases where the second iMRI session led to the conclusion of the surgery. Scan time was significantly different between these two groups of patients (i.e. two sessions or more than two sessions) in univariate analysis, but was not an independent factor in multivariate analysis.

In summary, univariate analysis revealed that non-eloquent area $(\mathrm{p}=0.016)$ and enhancing lesions $(\mathrm{p}=0.02)$ were predictive factors for complete resection (Tab. 2). Univariate analysis revealed that non-enhancing lesions and scan time were predictive factors for extended resection (Tab. 4), but the only independent factor for extended resection, as demonstrated in a Wald multivariate analysis, was non-enhancement (Tab. 5).

Table 5. Independent predictors of extended resection in a multivariate Wald test analysis. Variable(s) entered: age, gender, non-enhancement

\begin{tabular}{|c|c|c|c|c|c|c|c|}
\hline \multirow[t]{2}{*}{ Independent variables } & \multirow[t]{2}{*}{ B } & \multirow[t]{2}{*}{ S.E. } & \multirow[t]{2}{*}{ Wald } & \multirow[t]{2}{*}{$P$ value } & \multirow[t]{2}{*}{$\operatorname{Exp}(B)$} & \multicolumn{2}{|c|}{$95 \% \mathrm{Cl}$ for $\operatorname{Exp}(\mathrm{B})$} \\
\hline & & & & & & Lower & Upper \\
\hline Constant & 1.616 & 0.685 & 5.57 & 0.018 & 0.199 & & \\
\hline Age (years) & 0.005 & 0.015 & 0.107 & 0.744 & 1.005 & 0.976 & 1.035 \\
\hline Non-enhancement & 1.157 & 0.537 & 4.636 & 0.031 & 3.181 & 1.109 & 9.122 \\
\hline Gender & 0.597 & 0.681 & 0.77 & 0.38 & 1.817 & 0.479 & 6.901 \\
\hline
\end{tabular}




\section{Discussion}

The benefits of using the Polestar low-field iMRI system for achieving maximal resection of brain tumours have been reported by several groups, including ours [1, 2, 12-14].

Senft et al. [14] demonstrated particular benefits of the system for patients with contrast-enhancing tumours. Stereotactic biopsy using the Polestar is an accurate method for obtaining specimens with a high diagnostic yield [15]. An earlier version of the Polestar low-field iMRI system, the Polestar N20, was reported to have high sensitivity and specificity for residual tumour detection [16]. Other groups have also reported a significant benefit of other low-field iMRI systems in the removal of high-grade gliomas [17]. Claus et al. [9] reported a possible association between the extent of the resection of low-grade gliomas using low-field iMRI systems and a lower risk of recurrence or death.

In our study, we present in this cohort the advantages of the third generation low-field iMRI Polestar (N30), allowing for the use of standard surgical equipment, fully controlled and operated by the surgeon to increase the extent of the resection of non-enhancing lesions. The advantages of real-time imaging to localise and define lesion margins in the presence of dynamic changes in fluid and tissue compartments during resection were reported 20 years ago [18-19]. Several other centres have reported the usefulness of iMRI techniques for guidance in neurosurgical procedures $[2,9,20-25]$.

Emerging imaging technologies, as well as new intraoperative techniques, have expanded our ability to resect maximal amounts of tumour while preserving essential function. Stimulation mapping of language and motor pathways is known to contribute to the safe resection of intrinsic brain lesions [26, 27]. Additional techniques, including neuro-navigation, fluorescence-guided microsurgery using 5-aminolevulinic acid, iMRI, and ultrasonography, have all been applied to optimise the extent of resection in glioma patients.

Low-field iMRI can be used with other neurosurgical modalities: iMRI with 5-aminolevulinic acid-induced fluorescence provides synergistic effects in glioma resections [28]. Senft et al. [29] reported that the use of iMRI with neurophysiological monitoring in patients with gliomas located in eloquent brain areas increased the extent of the resection, without increasing the rate of neurological deficit.

On the basis of our experience up to 2012 [2], we present our more recent experience with the use of the third generation Polestar, an ultralow-field iMRI system, from February 2012 to the end of August 2018, in 73 craniotomies for intra-axial lesion tumour resection, almost half of which were non-enhancing lesions (35/73). The extent of the resection was expanded mainly for non-enhancing lesions (16/35 [46\%]) compared to enhancing lesions (only 8/38 [21\%]).

Our experience indicates that iMRI led to an extended resection in 24 of 73 patients with intra-axial brain lesions. Furthermore, iMRI allowed for further resection leading to complete lesion removal in 17 of 59 patients in whom complete lesion removal was intended, leading to a significant increase in complete resections achieved by our group. These findings correlate well with the values reported in previous studies [30]. In addition, we present a cohort of 13 patients in which the initial intent was to perform an incomplete resection and in which iMRI guidance led to additional resection in 6/13 (46\%) of the cases.

These findings suggest the usefulness of iMRI guidance for lesions located in eloquent brain regions for which the initial surgical intent was not necessarily to achieve a complete removal, but rather to maximise resection without endangering neurological stability.

In our study, low-grade gliomas were more frequent in cases where additional resection was performed ( $9 / 22$ compared to high-grade glioma [11/30] and miscellaneous pathologies [4/21] in which extended resection was less frequent). This raises questions about the benefit of iMRI in the resection of various pathologies. Some studies have included mixed pathology cohorts [9], whereas others have demonstrated a benefit in the resection of high-grade gliomas.

Although dividing the patients into three groups is a potential limitation of our study, the purpose of the study was to evaluate the utility of the Polestar N30 to achieve resection control of non-enhancing lesions, regardless of the WHO grading, which means both low-grade and high-grade non-enhancing gliomas.

In our study, multivariate analysis to compare the cases in which iMRI did not lead to further resection to cases in which it enabled maximal extent of resection (without considering the amount of resection) revealed a significant difference in regard to the lesion enhancement properties.

Non-enhancing lesions of all types were three times more likely to require additional resection after iMRI resection control. These findings support our previous experience [2] and point to a possible advantage for all non-enhancing lesions, including non-enhancing high-grade gliomas, which were more common $(12 / 30)$ in this cohort. No correlation was detected between recurrent tumours in cases in which additional resection was performed.

Imaging duration and extended surgery duration may be a reason for surgeons not to use intraoperative imaging modalities. With the development of user-friendly ultralow-field systems, the increase in surgery duration has become quite reasonable, with a commonly cited mean of $1 \mathrm{~h}$ extended overall surgery time $[17,31,32]$. This finding is in accordance with a mean surgical time prolongation of $60 \mathrm{~min}$ for all patients in whom more than two sessions were performed in the current cohort.

In our opinion, this is an acceptable delay in surgery completion. Furthermore, in $75 \%$ of cases in which iMRI led to complete resection, three scan sessions sufficed, which indicates how infrequently more than three scan sessions were required. 
Carabenciov et al. [33] stated that in the context of the new WHO classification system, all low-grade gliomas must have an IDH mutation, with or without a $1 \mathrm{p} / 19 \mathrm{q}$ co-deletion. Upon discovery of the tumour, maximal safe surgical resection is the most appropriate first step due to the current inability to differentiate between IDH mutant and IDH wild-type tumours by imaging alone.

Hervey-Jumper et al. [34] reported that low-grade gliomas have 5 - and 10 -year survival rates of $97 \%$ and $91 \%$ respectively, when the extent of resection is greater than $90 \%$. Duffau et al. [27] demonstrated the prolonged impact of supratotal resection on malignant transformation of low-grade gliomas. These findings emphasise the importance of maximising the extent of resection of non-enhancing low-grade tumours. Up to $50 \%$ of anaplastic gliomas (grade III) are non-enhancing or slurred enhancing lesions, as demonstrated in our cohort as well. Furthermore, Kawaguchi et al. [35] showed in a retrospective study of 124 consecutive patients with newly diagnosed grade III gliomas that strict surgical removal is important to improve the prognosis of patients with grade III gliomas, especially for tumours with the IDH $1 / 2$ mutation without $1 \mathrm{p} / 19 \mathrm{q}$ co-deletions.

Leroy et al. [25] reported that iMRI was more useful for non- or minimally-enhancing tumours, as shown in our previous cohort [2]. A retrospective multicentre series of 288 cases of low-grade gliomas indicated an independent association of high-field (1.5-T) iMRI guidance with the rate of gross total resections after surgery, without an accumulated risk of neurological deficits [36]. In a recent analysis, Swinney et al. [37] reviewed 12 studies that included 804 primary operations and 238 extended resections based on iMRI findings. Use of iMRI led to extended tumour resection in 13.3-54.8\% of patients (mean $37.3 \%$ ). Stratification by tumour type showed that additional resection was performed on average in 39.1\% of glioma resections (range 13.3-70.0\%), 23.5\% of pituitary tumour resections (range 13.3-33.7\%), and $35.0 \%$ of nonspecific tumour resections (range 17.5-40\%). These findings are similar to the extent of resections performed in our previous cohort [2] and the current study.

There are very few recent reports on low-field iMRI. Some previous studies demonstrated that low-field iMRI influenced intraoperative decision-making and improved brain tumour resection $[9,14,17]$. Other intraoperative modalities, such as fluorescence-guided surgery and ultrasound-guided surgery, may be easier to operate and less time-consuming, and may improve the resection of low-grade gliomas [12]. Intraoperative tumour visualisation with 5 -aminolevulinic acid (5-ALA) induced protoporphyrin IX (PpIX) fluorescence is widely applied for improved resection of high-grade gliomas: Stummer et al. [6] demonstrated in a series of 139 patients enrolled in a randomised controlled multicentre phase III trial that complete resection of enhancing tumours was increased from $36 \%$ (white light) to 65\% (5-ALA) and progression-free survival at six months increased from $21 \%$ (white light) to $41 \%$
(5-ALA). Recently, visible fluorescence of low-grade gliomas was reported as well $[38,39]$.

Jaber et al. [40] reported that only $15.9 \%$ of 82 WHO grade II tumours revealed intraoperative PpIX fluorescence compared to $83.3 \%$ of enhancing grade III tumours. Goryaynov et al. [38], however, showed a markedly higher rate of up to $52 \%$ visible fluorescence in a series of low-grade gliomas. In addition, they demonstrated that the use of antiepileptic drugs reduces the presence of visible fluorescence in low-grade gliomas. Widhalm et al. [39] showed the benefit of quantitative PpIX analysis in diffusely infiltrating low-grade glioma.

Stepp and Stummer [41] in a recent review summarised the valuable diagnostic options of 5-ALA in the management of malignant gliomas and also explored biological boosters of selective PpIX accumulation for the therapeutic use of 5-ALA. In addition, they looked at surgical guidance tools other than 5-ALA fluorescence-guided surgery.

Sastry et al. [42] reviewed the utility of intraoperative ultrasound since 1982 for brain tumour resection: intraoperative navigation, assessment of extent of resection, and brain shift monitoring and compensation. Selbekk et al. [43] reported techniques to identify and reduce image artefacts in ultrasound images that may occur during brain tumour surgery.

Taking into consideration the advancement of these other modalities raises the question of the need to sustain the low-field iMRI modality. The Polestar N30 is no longer manufactured! Is this the end of the era for low-field iMRI or should we make an effort to preserve this modality, especially for non-enhancing lesions?

The role of iMRI for enhancing and especially for non-enhancing gliomas in the era of fluorescence-guided surgery and advanced ultrasound modalities needs to be better defined.

Our data supports the need to preserve iMRI for extended resection, especially for non-enhancing brain lesions. We believe that it would be wise to preserve this technology for resection control. In contrast to low-field iMRI, however, high-field iMRI has several other benefits, such as the ability to create diffuse tensor images [44], laser-guided surgery [45], and focused ultrasound procedures, including research benefits such as opening of the blood brain barrier [46, 47]. It may be advisable to use high-magnetic power field iMRI for extended resection, taking into consideration its benefits and our findings that support preservation of the modality.

\section{Conclusions}

Intraoperative MRI guidance markedly improved the surgeon's ability to maximise the extent of resection in our series of 73 patients with intra-axial lesions.

The iMRI system allowed for additional resection in $32.8 \%$ of all cases and for complete resection in $28 \%$ of the cases when complete resections was intended. Additionally, iMRI allowed for extended resection in $46.1 \%$ of patients for whom 
the intent was to perform an incomplete resection. The extent of resection was extended mainly for non-enhancing lesions, $16 / 35$ as opposed to only $8 / 38$ for enhancing lesions.

Non-enhancing lesions were the only independent variable predicting use of iMRI for extended resection. The procedure can be performed under electrophysiological monitoring using magnetic-resonance compatible electrodes, allowing for preserved function with a reasonable mean surgery time elongation of only 60 minutes.

Our results provide additional support for the benefits of this technology for achieving a maximal resection, especially in patients with non-enhancing intra-axial brain tumours.

Conflict of interest: All authors declare no conflict of interest. The authors have no personal financial or institutional interest in any of the drugs, materials, or devices described in this article. Funding: This research received no specific grant from any funding agency in the public, commercial, or not-for-profit sectors. Contributorship statement: $L U, Z Z$, and $Z R C$ conducted the study, performed the surgical procedures, reviewed all the radiological images, interpreted the data, and edited the manuscript.

- RH and NK collected and interpreted the data.

- $M H, M A, Z F, J Z, A W$, and RS performed the surgical procedures or were part of the decision-making process.

- All authors read and approved the final manuscript.

Ethical permission: This study was approved by the Sheba Medical Centre ethics committee, IRB no: 6770-19-SMC.

\section{References}

1. Hadani M, Spiegelman R, Feldman Z, et al. Novel, compact, intraoperative magnetic resonance imaging-guided system for conventional neurosurgical operating rooms. Neurosurgery. 2001; 48(4): 799-807; discussion 807, doi: 10.1097/00006123-200104000-00021, indexed in Pubmed: 11322440.

2. Livne O, Harel R, Hadani M, et al. Intraoperative magnetic resonance imaging for resection of intra-axial brain lesions: a decade of experience using low-field magnetic resonance imaging, Polestar N-10, 20, 30 systems. World Neurosurg. 2014; 82(5): 770776, doi: 10.1016/j.wneu.2014.02.004, indexed in Pubmed: 24518885.

3. Hess KR. Extent of resection as a prognostic variable in the treatment of gliomas. J Neurooncol. 1999; 42(3): 227-231, doi: 10.1023/a:1006118018770, indexed in Pubmed: 10433106.

4. Keles GE, Anderson B, Berger MS. The effect of extent of resection on time to tumor progression and survival in patients with glioblastoma multiforme of the cerebral hemisphere. Surg Neurol. 1999; 52(4): 371-379, doi: 10.1016/s0090-3019(99)00103-2, indexed in Pubmed: 10555843.

5. Lacroix M, Abi-Said D, Fourney DR, et al. A multivariate analysis of 416 patients with glioblastoma multiforme: prognosis, extent of resection, and survival. J Neurosurg. 2001; 95(2): 190-198, doi: 10.3171/ jns.2001.95.2.0190, indexed in Pubmed: 11780887.

6. Stummer W, Pichlmeier U, Meinel T, et al. ALA-Glioma Study Group. Fluorescence-guided surgery with 5-aminolevulinic acid for resection of malignant glioma: a randomised controlled multicentre phase
III trial. Lancet Oncol. 2006; 7(5): 392-401, doi: 10.1016/S14702045(06)70665-9, indexed in Pubmed: 16648043.

7. Sanai N, Berger MS. Glioma extent of resection and its impact on patient outcome. Neurosurgery. 2008; 62(4): 753-64; discussion 264, doi: 10.1227/01.neu.0000318159.21731.cf, indexed in Pubmed: 18496181.

8. Senft C, Bink A, Heckelmann M, et al. Glioma extent of resection and ultra-low-field iMRI: interim analysis of a prospective randomized trial. Acta Neurochir Suppl. 2011; 109: 49-53, doi: 10.1007/978-3-211 99651-5_8, indexed in Pubmed: 20960320.

9. Claus EB, Horlacher A, Hsu L, et al. Survival rates in patients with low-grade glioma after intraoperative magnetic resonance image guidance. Cancer. 2005; 103(6): 1227-1233, doi: 10.1002/cncr.20867, indexed in Pubmed: 15690327.

10. Smith JS, Chang EF, Lamborn KR, et al. Role of extent of resection in the long-term outcome of low-grade hemispheric gliomas. J Clin Oncol. 2008; 26(8): 1338-1345, doi: 10.1200/JC0.2007.13.9337, indexed in Pubmed: 18323558.

11. Snyder LA, Wolf AB, Oppenlander ME, et al. The impact of extent of resection on malignant transformation of pure oligodendrogliomas. J Neurosurg. 2014; 120(2): 309-314, doi: 10.3171/2013.10. JNS13368, indexed in Pubmed: 24313617.

12. D'Amico RS, Englander ZK, Canoll P, et al. Extent of Resection in Glioma-A Review of the Cutting Edge. World Neurosurg. 2017; 103: 538-549, doi: 10.1016/j.wneu.2017.04.041, indexed in Pubmed: 28427971.

13. Schulder M, Carmel PW. Intraoperative magnetic resonance imaging: impact on brain tumor surgery. Cancer Control. 2003; 10(2): 115-124, doi: 10.1177/107327480301000203, indexed in Pubmed: 12712006 .

14. Senft C, Seifert V, Hermann E, et al. Usefulness of intraoperative ultra low-field magnetic resonance imaging in glioma surgery. Neurosurgery. 2008; 63(4 Suppl 2): 257-66; discussion 266, doi: 10.1227/01. NEU.0000313624.77452.3C, indexed in Pubmed: 18981831.

15. Quinn J, Spiro D, Schulder M. Stereotactic brain biopsy with a low-field intraoperative magnetic resonance imager. Neurosurgery. 2011; 68(1 Suppl Operative): 217-24; discussion 224, doi: 10.1227/ NEU.0b013e31820826c2, indexed in Pubmed: 21206306.

16. Hirschl RA, Wilson J, Miller B, et al. The predictive value of low-field strength magnetic resonance imaging for intraoperative residual tumor detection. Clinical article. J Neurosurg. 2009; 111(2): 252-257, doi: 10.3171/2008.9.JNS08729, indexed in Pubmed: 19199455.

17. Knauth M, Wirtz CR, Tronnier VM, et al. Intraoperative MR imaging increases the extent of tumor resection in patients with high-grade gliomas. AJNR Am J Neuroradiol. 1999; 20(9): 1642-1646, indexed in Pubmed: 10543634.

18. Black PM, Moriarty T, Alexander E, et al. Development and implementation of intraoperative magnetic resonance imaging and its neurosurgical applications. Neurosurgery. 1997; 41(4): 831-42; discussion 842, doi: 10.1097/00006123-199710000-00013, indexed in Pubmed: 9316044.

19. Seifert V. Intraoperative MRI in neurosurgery: technical overkill or the future of brain surgery? Neurol India. 2003; 51(3): 329-332, indexed in Pubmed: 14652431.

20. Bohinski R, Warnick R, Gaskill-Shipley M, et al. Intraoperative Magnetic Resonance Imaging to Determine the Extent of Resection of Pituitary Macroadenomas during Transsphenoidal Microsurgery. Neurosurgery. 2001; 49(5): 1133-1144, doi: 10.1227/00006123200111000-00023. 
21. Nimsky C, von Keller B, Ganslandt O, et al. Intraoperative high-field magnetic resonance imaging in transsphenoidal surgery of hormonally inactive pituitary macroadenomas. Neurosurgery. 2006; 59(1): 10514; discussion 105, doi: 10.1227/01.NEU.0000219198.38423.1E, indexed in Pubmed: 16823306.

22. Gerlach R, du Mesnil de Rochemont R, Gasser T, et al. Feasibility of Polestar N2O, an ultra-low-field intraoperative magnetic resonance imaging system in resection control of pituitary macroadenomas: lessons learned from the first 40 cases. Neurosurgery. 2008; 63(2): 272-84; discussion 284, doi: 10.1227/01.NEU.0000312362.63693.78, indexed in Pubmed: 18797357.

23. Baumann F, Schmid C, Bernays RL. Intraoperative magnetic resonance imaging-guided transsphenoidal surgery for giant pituitary adenomas. Neurosurg Rev. 2010; 33(1): 83-90, doi: 10.1007/s10143-0090230-4, indexed in Pubmed: 19823884.

24. Olubiyi Ol, Ozdemir A, Incekara F, et al. Intraoperative Magnetic Resonance Imaging in Intracranial Glioma Resection: A Single-Center, Retrospective Blinded Volumetric Study. World Neurosurg. 2015; 84(2): 528-536, doi: 10.1016/j.wneu.2015.04.044, indexed in Pubmed: 25937354.

25. Leroy HA, Delmaire C, Le Rhun E, et al. High-field intraoperative MRI and glioma surgery: results after the first 100 consecutive patients. Acta Neurochir (Wien). 2019; 161(7): 1467-1474, doi: 10.1007/ s00701-019-03920-6, indexed in Pubmed: 31073784.

26. Duffau H. A new philosophy in surgery for diffuse low-grade glioma (DLGG): oncological and functional outcomes. Neurochirurgie. 2013; 59(1): 2-8, doi: 10.1016/j.neuchi.2012.11.001, indexed in Pubmed: 23410764.

27. Duffau H. Long-term outcomes after supratotal resection of diffuse low-grade gliomas: a consecutive series with 11-year follow-up. Acta Neurochir (Wien). 2016; 158(1): 51-58, doi: 10.1007/s00701-0152621-3, indexed in Pubmed: 26530708.

28. Tsugu A, Ishizaka H, Mizokami Y, et al. Impact of the combination of 5-aminolevulinic acid-induced fluorescence with intraoperative magnetic resonance imaging-guided surgery for glioma. World Neurosurg. 2011; 76(1-2): 120-127, doi: 10.1016/j.wneu.2011.02.005, indexed in Pubmed: 21839963.

29. Senft C, Forster MT, Bink A, et al. Optimizing the extent of resection in eloquently located gliomas by combining intraoperative MRI guidance with intraoperative neurophysiological monitoring. J Neurooncol. 2012; 109(1): 81-90, doi: 10.1007/s11060-012-0864-x, indexed in Pubmed: 22528791.

30. Berger MS, Deliganis AV, Dobbins J, et al. The effect of extent of resection on recurrence in patients with low grade cerebral hemisphere gliomas. Cancer. 1994; 74(6): 17841791, doi: 10.1002/1097-0142(19940915)74:6<1784::aid-cncr2820740622>3.0.co;2-d, indexed in Pubmed: 8082081.

31. Archer DP, McTaggart Cowan RA, Falkenstein RJ, et al. Intraoperative mobile magnetic resonance imaging for craniotomy lengthens the procedure but does not increase morbidity. Can J Anaesth. 2002; 49(4): 420-426, doi: 10.1007/BF03017334, indexed in Pubmed: 11927485.

32. Makary M, Chiocca EA, Erminy N, et al. Clinical and economic outcomes of low-field intraoperative MRI-guided tumor resection neurosurgery. J Magn Reson Imaging. 2011; 34(5): 1022-1030, doi: 10.1002/ jmri.22739, indexed in Pubmed: 22002753.

33. Carabenciov ID, Buckner JC. Controversies in the Therapy of Low-Grade Gliomas. Curr Treat Options Oncol. 2019; 20(4): 25, doi: 10.1007/ s11864-019-0625-6, indexed in Pubmed: 30874903.
34. Hervey-Jumper SL, Berger MS. Role of surgical resection in low- and high-grade gliomas. Curr Treat Options Neurol. 2014; 16(4): 284, doi: 10.1007/s11940-014-0284-7, indexed in Pubmed: 24595756.

35. Kawaguchi T, Sonoda Y, Shibahara I, et al. Impact of gross total resection in patients with WHO grade III glioma harboring the IDH 1/2 mutation without the 1p/19q co-deletion. J Neurooncol. 2016; 129(3): 505-514, doi: 10.1007/s11060-016-2201-2, indexed in Pubmed: 27401154.

36. Coburger J, Merkel A, Scherer M, et al. Low-grade Glioma Surgery in Intraoperative Magnetic Resonance Imaging: Results of a Multicenter Retrospective Assessment of the German Study Group for Intraoperative Magnetic Resonance Imaging. Neurosurgery. 2016; 78(6): 775-786, doi: 10.1227/NEU.0000000000001081, indexed in Pubmed: 26516822.

37. Swinney C, Li A, Bhatti I, et al. Optimization of tumor resection with intra-operative magnetic resonance imaging. J Clin Neurosci. 2016; 34: 11 14, doi: 10.1016/j.jocn.2016.05.030, indexed in Pubmed: 27469412.

38. Goryaynov SA, Widhalm G, Goldberg MF, et al. The Role of 5-ALA in Low-Grade Gliomas and the Influence of Antiepileptic Drugs on Intraoperative Fluorescence. Front Oncol. 2019; 9: 423, doi: 10.3389/ fonc.2019.00423, indexed in Pubmed: 31192128.

39. Widhalm G, Olson J, Weller J, et al. The value of visible 5-ALA fluorescence and quantitative protoporphyrin IX analysis for improved surgery of suspected low-grade gliomas. J Neurosurg. 2019 [Epub ahead of print]: 1-10, doi: 10.3171/2019.1.JNS182614, indexed in Pubmed: 31075771.

40. Jaber M, Wölfer J, Ewelt C, et al. The Value of 5-Aminolevulinic Acid in Low-grade Gliomas and High-grade Gliomas Lacking Glioblastoma Imaging Features: An Analysis Based on Fluorescence, Magnetic Resonance Imaging, 18F-Fluoroethyl Tyrosine Positron Emission Tomography, and Tumor Molecular Factors. Neurosurgery. 2016; 78(3): 401-11; discussion 411, doi: 10.1227/NEU.0000000000001020, indexed in Pubmed: 26366972.

41. Stepp H, Stummer W. 5-ALA in the management of malignant glioma. Lasers Surg Med. 2018; 50(5): 399-419, doi: 10.1002/Ism.22933, indexed in Pubmed: 29737540.

42. Sastry R, Bi WL, Pieper S, et al. Applications of Ultrasound in the Resection of Brain Tumors. J Neuroimaging. 2017; 27(1): 5-15, doi: 10.1111/jon.12382, indexed in Pubmed: 27541694.

43. Selbekk T, Jakola AS, Solheim 0, et al. Ultrasound imaging in neurosurgery: approaches to minimize surgically induced image artefacts for improved resection control. Acta Neurochir (Wien). 2013; 155(6): 973-980, doi: 10.1007/s00701-013-1647-7, indexed in Pubmed: 23459867.

44. Elliott $\mathrm{CA}$, Danyluk H, Aronyk KE, et al. Intraoperative acquisition of DTI in cranial neurosurgery: readout-segmented DTI versus standard single-shot DTI. J Neurosurg. 2019 [Epub ahead of print]: 1-10, doi: 10.3171/2019.5.JNS19890, indexed in Pubmed: 31419798.

45. Lagman C, Chung LK, Pelargos PE, et al. Laser neurosurgery: A systematic analysis of magnetic resonance-guided laser interstitial thermal therapies. J Clin Neurosci. 2017; 36: 20-26, doi: 10.1016/j. jocn.2016.10.019, indexed in Pubmed: 27838155.

46. Halpern CH, Santini V, Lipsman N, et al. A Randomized Trial of Focused Ultrasound Thalamotomy for Essential Tremor. N Engl J Med. 2016; 375(8): 730-739, doi: 10.1056/NEJMoa1600159, indexed in Pubmed: 27557301.

47. Aryal M, Papademetriou I, Zhang YZ, et al. MRI Monitoring and Quantification of Ultrasound-Mediated Delivery of Liposomes Dually Labeled with Gadolinium and Fluorophore through the Blood-Brain Barrier. Ultrasound Med Biol. 2019; 45(7): 1733-1742, doi: 10.1016/j.ultrasmedbio.2019.02.024, indexed in Pubmed: 31010598. 